PDFlib PLOP: PDF Linearization, Optimization, Protection

Page inserted by evaluation version www.pdflib.com - sales@pdflib.com 


\title{
Proteomic Patterns of Cultured Breast Cancer Cells and Epithelial Mammary Cells
}

\author{
IDA PUCCI-MINAFRA,${ }^{a}$ SIMONA FONTANA, PATRIZIA CANCEMI, \\ GIUSEPPINA ALAIMO, AND SALVATORE MINAFRA \\ University of Palermo, Center of Experimental Oncobiology, Department of Cell Biology \\ and Development, Institute of Histology and Embryology, Palermo, Italy
}

\begin{abstract}
Breast cancer is one of the leading causes of death from cancer among women in western countries. The different types of breast cancer are grouped into invasive and noninvasive forms. Among the invasive types, ductal infiltrating carcinoma (DIC) is the most common and aggressive form. Using an in vitro model consisting of a DIC-derived cell line (8701-BC) and a nontumoral mammary epithelial cell line (HB2), we used the proteomics approach to search for homology and differences in protein expression patterns between tumoral and nontumoral phenotypes. Within an analysis window comprising 1,750 discernible spots we have currently catalogued 140 protein spots of potential interest. Fifty-eight of them were identified by gel matching with reference maps, immunodetection, or $\mathbf{N}$-terminal microsequencing and classified into four functional groups. Twelve proteins were found differentially expressed in two cell lines: four were uniquely present in the neoplastic cell proteome and eight in epithelial cells. In addition, 53 proteins displayed different relative expression levels between the two cell lines, that is, 44 were more elevated in cancer cells and 9 in $\mathrm{HB} 2$ cells. Among proteins with greater relative abundance in cancer cells we identified glycolytic enzymes (or their isoforms), which may indicate that the known metabolic dysregulation in cancer can reflect oncogenic-related defects of glycolytic gene expression.
\end{abstract}

KEYWORDS: proteomics; breast cancer; ductal infiltrating carcinoma

\section{INTRODUCTION}

Cancer of the mammary gland is still one of the leading causes of death from tumor among women in western countries. However, breast cancer is not a single disease but includes several different forms grouped as invasive and noninvasive types. Among the invasive histotypes, ductal infiltrating carcinoma (DIC) is the most common and aggressive form of breast cancer. ${ }^{1}$ It usually has a poor prognosis and represents the standard histotype with which the other less frequent subtypes (i.e., papillary, mucinous-colloid, tubular, medullary carcinomas) are compared. Carcinomas are potentially malignant tumors of epithelial origin, which, once genetically

Address for correspondence: Prof. Ida Pucci Minafra, Center of Experimental Oncobiology, University of Palermo, Viale delle Scienze, 90128 Palermo, Italy. Voice/fax: 3991424903. idapucci@unipa.it

Ann. N.Y. Acad. Sci. 963: 122-139 (2002). @ 2002 New York Academy of Sciences. 
"initiated," grow, progress, invade, and spread in the body with a common phenomenology regardless of their individual derivation.

The major cellular changes involved in the conversion of a normal to a malignant breast are the progressive loss of the stationary epithelial phenotype (consisting of polarized morphology and specific cell-cell and cell-matrix adhesion systems) and the acquisition of a mesenchymal-like phenotype, correlated with the ability to migrate and invade surrounding and distant tissues, even under conditions of a low oxygen supply. These aspects are related to multiple gene defects, whose functional network is hard to understand from a global point of view.

According to recent estimation, the human genome encodes at least 30,000 40,000 structural genes. ${ }^{2,3}$ However, due to posttranscriptional and posttranslational modifications, the number of proteins expressed by cells may be several times higher. In addition, posttranslational modifications may be instrumental in altering or adding functions to certain proteins and their oligomeric forms. Therefore, detecting protein profiles, directed towards knowledge of both the identity and the relative quantity of proteins (and their isoforms) in tumors, is the challenge of the postgenomic era. Comparative profiling of paired tumoral and normal counterparts may provide substantial information on qualitative and quantitative modifications of cancer-associated gene expression, which in turn can be fundamental in understanding molecular mechanisms involved in cancer development. The proteomic strategy, based on a high-resolution two-dimensional immobilized $\mathrm{pH}$ gradient (2D-IPG) and related techniques (computer-assisted image analysis, microsequencing, and immune detection), is a powerful tool for the separation, detection, and analysis of a large number of proteins on the same preparation, without prior knowledge of their identity.

The study of protein expression in primary human breast cancer (as for most solid tumors), however, is difficult because breast tumors may contain several different cell types, other than carcinoma cells, such as additional epithelial cell types, stromal cells, and infiltrating lymphocytes. On the other hand, laser-capture microdissected sections ${ }^{4}$ may contain large amounts of proteins resident in the extracellular interstitium as albumin, globulins, and other serum proteins. Therefore, several laboratories, including ours, have developed an appropriate in vitro model of normal and neoplastic mammary cells for biological and molecular assays ${ }^{5-8}$ including the recent approach on cDNA microarray ${ }^{9}$ and proteomics. ${ }^{10}$

Among the breast cancer cell lines described to date in the literature, $8701-\mathrm{BC}^{5}$ deserves particular interest, because it was established from a primary DIC, that is, before clonal selection of the metastatic process. As a nontumoral reference cell line, we used HB2 cells ${ }^{7}$ that maintain in culture the lumenal mammary phenotype, which represents the most common target of mammary cancer.

On protein separation by $2 \mathrm{D}-\mathrm{IPG}$ and computer processing of the $2 \mathrm{D}$ gels, we searched for qualitative (presence/absence) and quantitative (relative abundance) differences in protein profiles to identify relevant differences and homologies between the two representative cell lines.

Within an analysis window comprising about 1,750 discernible spots, we have currently catalogued 140 proteins of potential interest, 58 of which were identified by gel matching, immunodetection, or $\mathrm{N}$-terminal microsequencing. Twelve proteins were differentially expressed in the two cell lines: four were expressed uniquely by neoplastic cells and eight by epithelial cells. In addition, 53 proteins of 140 
selected in the map displayed different relative quantities, namely, 44 proteins were more elevated in cancer cells and 9 in HB2 cells.

Interestingly, among proteins with higher relative expression levels in cancer cells (at least 1.5- to 3-fold) we identified several glycolytic enzymes and their isoforms. This finding is of interest, because the "Warburg effect" (enhanced anaerobic metabolism in cancer) has long been known, but this is the first report of increased levels of these enzymes in cancer cells at the proteomic level. Therefore, due to their easy detection on the 2D map, the present approach could have great clinical application.

\section{MATERIAL AND METHODS}

\section{Cell Culture}

Cells were grown in the appropriate culture medium supplemented with $10 \%$ fetal calf serum (FCS; GIBCO) and antibiotics (100 units/ml penicillin and $100 \mu \mathrm{g} /$ $\mathrm{ml}$ streptomycin) in a humidified incubator with $3 \% \mathrm{CO}_{2}$ in air at $37^{\circ} \mathrm{C}$. The culture medium was RPMI 1640 for the 8701-BC 5 and Dulbecco's modified Eagle's medium (DMEM) supplemented with hydrocortisone $(5 \mu \mathrm{g} / \mathrm{ml})$ and insulin $(10 \mu \mathrm{g} / \mathrm{ml})$ for the HB2 human mammary epithelial cell line. ${ }^{7}$

\section{Sample Preparations}

Cells were grown to confluence and then incubated with three changes of serumfree medium. After washing with ice-cold phosphate-buffered saline solution, they were carefully scraped and incubated in ice for 30 minutes with RIPA buffer $(50 \mathrm{mM}$ Tris $\mathrm{pH} 7.5,0.1 \%$ nonidet $\mathrm{P}-40,0.1 \%$ deoxycholate, $150 \mathrm{mM} \mathrm{NaCl}, 4 \mathrm{mM}$ EDTA, and a mixture of protease inhibitors, $0.01 \%$ aprotinin, $10 \mathrm{mM}$ sodium pyrophosphate, $2 \mathrm{mM}$ sodium orthovanadate, and $1 \mathrm{mM}$ PMSF). The total cellular lysate was centrifuged at 14,000 rpm for 8 minutes to clear cell debris, and the supernatant was dialyzed against ultrapure distilled water, lyophilized, and stored at $-80^{\circ} \mathrm{C}$ until analysis. Protein concentration in the cellular extracts was determined using the Breadford method.

\section{Two-Dimensional Gel Electrophoresis}

Aliquots of dried cell lysate were solubilized in buffer containing $8 \mathrm{M}$ urea, $4 \%$ CHAPS, $40 \mathrm{mM}$ Tris, $65 \mathrm{mM}$ DTE (1,4-dithioerythritol), and trace amounts of bromophenol blue.

The first dimensional separation was performed at $20^{\circ} \mathrm{C}$ on commercial sigmoidal immobilized $\mathrm{pH}$ gradient strips (IPG) $18 \mathrm{~cm}$ long, $\mathrm{pH} 3.5-10$ (Pharmacia), essentially as described by Görg et al. ${ }^{11}$ and Bjellqvist et al. ${ }^{12}$ Strips were rehydrated in $8 \mathrm{M}$ urea, 2\% CHAPS, $10 \mathrm{mM}$ DTE, and $0.5 \%$ carrier ampholytes (resolyte 3.510). Aliquots of $45 \mu \mathrm{g}$ (analytical gels) or $1.5 \mathrm{mg}$ (preparative gels) total proteins were applied to the gel strip. Isoelectrofocusing was carried out by linearly increasing voltage from 200-3,500 V during the first 4.5 hours, after which focusing was continued at $8,000 \mathrm{~V}$ for 8 hours. After the run, the IPG strips were equilibrated with a solution containing $6 \mathrm{M}$ urea, $30 \%$ glycerol, $2 \% \mathrm{SDS}, 0.05 \mathrm{M}$ Tris- $\mathrm{HCl} \mathrm{pH} \mathrm{6.8,} \mathrm{and}$ $2 \%$ DTE for 12 minutes to resolubilze proteins and reduce disulfur bonds. The -SH 
groups were then blocked by substituting the DTE with $2.5 \%$ iodoacetamide in the equilibrating buffer.

The focused proteins were then separated on $9-16 \%$ linear gradient polyacrylamide gels (SDS-PAGE) with a constant current of $40 \mathrm{~mA} / \mathrm{gel}$ at $10^{\circ} \mathrm{C}$. Gels were stained with ammoniacal silver nitrate, as described by Hochstrasser et al. ${ }^{13}$

\section{Image Acquisition and Data Analysis}

Silver-stained gels were digitized using a computing densitometer and processed with a Melanie 3 computer system (GeneBio). Gel calibration was carried out on the basis of internal standard with the support of the ExPaSy molecular biology server. Quantitative variations of proteins were expressed as volumes of spots (i.e., integration of optical density over the spot area). To correct for differences in gel staining, relative volumes to the sum of the volume of all spots in each gel were calculated with the Melanie system and plotted with the Excel program. For figure presentation, images were transferred to Adobe Photoshop and PowerPoint programs.

\section{Protein Microsequencing}

$\mathrm{N}$-terminal microsequencing was performed by automated Edman degradation in an Applied Biosystem protein sequenator (Procise, Perkin-Elmer) on protein samples electrotransferred onto polyvinylidene difluoride membranes (ImmobilonPTM, Millipore). The SWISS-PROT (ScanProsite) and EMBL (Bic 2) databases were used for online sequence similarity search at http://expasy.hcuge.ch/.

\section{RESULTS}

\section{The In Vitro Model}

As an in vitro model for present research, we used two representative cell lines: the 8701-BC, derived from a primary ductal infiltrating carcinoma, ${ }^{5}$ which shows invasive behavior both in vivo and in vitro, ${ }^{14-16}$ and the nontumorigenic HB2 cell line, derived from immortalized human lumenal mammary epithelial cells, ${ }^{7}$ kindly supplied by Dr. J. Taylor-Papadimitriou (Imperial Cancer Research Fund, London, United Kingdom) and presently cultivated in our laboratory.

FIGURE 1 shows representative phase-contrast micrographs of the two cell cultures at the following time of growth: 24 hours, 48 hours, 72 hours, and 1 week after seeding. As can be observed in the micrographs from A to D, the neoplastic cells display an apolarized morphology with irregular profiles; they spread over the substrate with emission of spikes and once they reach confluence, they overgrow, forming typical domes (FIG. 1D). On the contrary, HB2 cells (FIG. 1E-H) maintain in vitro the morphology of epithelial cells, being polarized and responsive to contact inhibition.

\section{Proteomic Profiles of the 8701-BC and HB2 Cell Lines}

Each cell culture was grown until confluence, deprived of serum, and then processed for lysis and two-dimensional-IPG separation, as described in Material and Methods. Figures 2 and 3 show the miniature of two representative 2D maps of the 

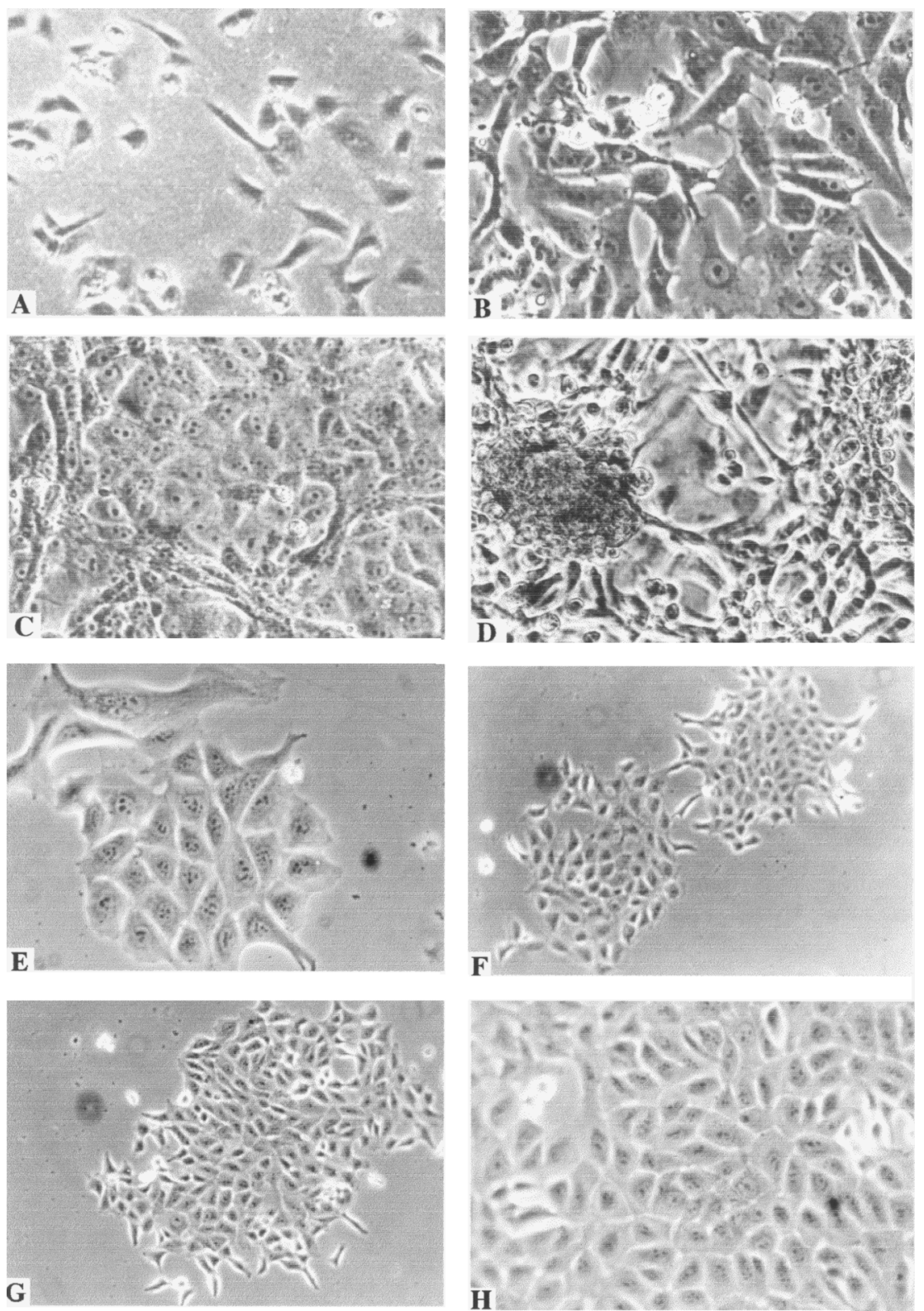

FIGURE 1. Phase contrast micrographs of 8701-BC cells (A, B, C, and D) and HB2 cells (E, F, G, and $\mathbf{H}$ ) at different times from seeding: 24 hours, 48 hours, 72 hours, and 1 week, respectively. Note the different pattern of cell growth and morphology: HB2 cells maintain in culture a polarized morphology and contact inhibition, differently from neoplastic cells, which show apolarized morphology and form multilayered "domes" in the absence of contact inhibition. The two cell lines were confluent at the time of the experiments $(600 \times)$. 


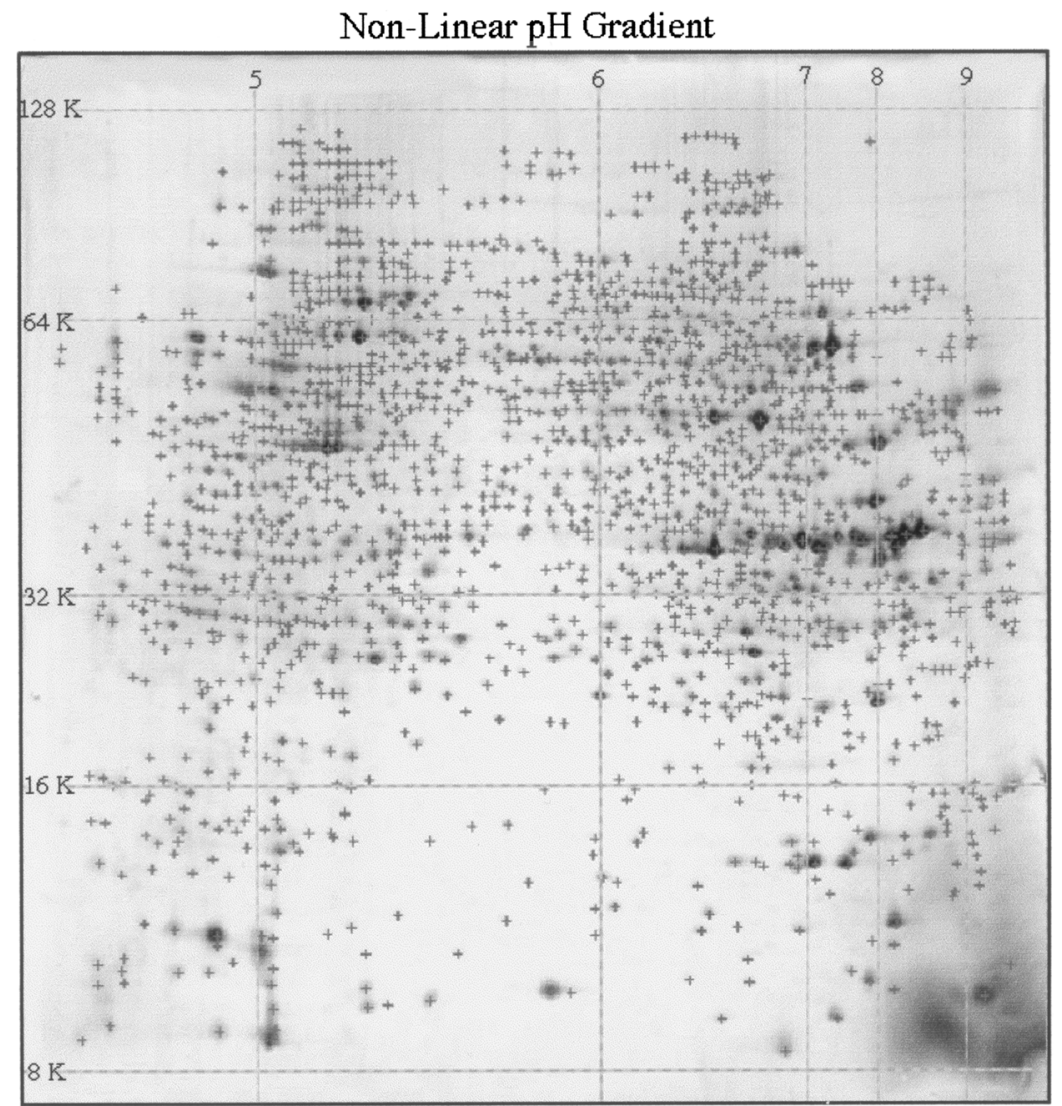

FIGURE 2. Representative two-dimensional electrophoretogram of proteins in 8701$3 \mathrm{C}$ cell line. Crosses and grid indicating $\mathrm{p} I$ and $\mathrm{Mr}$ were automatically assigned by the Melanie system.

8701-BC and HB2 cells, respectively. The crosses on the spots as well as the grid indicating $\mathrm{p} I$ and $\mathrm{Mr}$ were assigned by the Melanie system after cleaning out the background from maps. The number of spots was 1,747 in cancer cells and 1,753 in HB2 cells.

\section{Protein Identification and Clustering in the 8701-BC Map}

Protein identification in the 8701-BC map was performed by gel matching, immunodetection, or $\mathrm{N}$-terminal microsequencing, as described in Material and Methods. Currently, 58 proteins (including isoforms) were identified in the map and grouped according to their major functions. The first group includes cytoskeletal proteins, the second group folding, chaperons, and heat-shock proteins, the third 


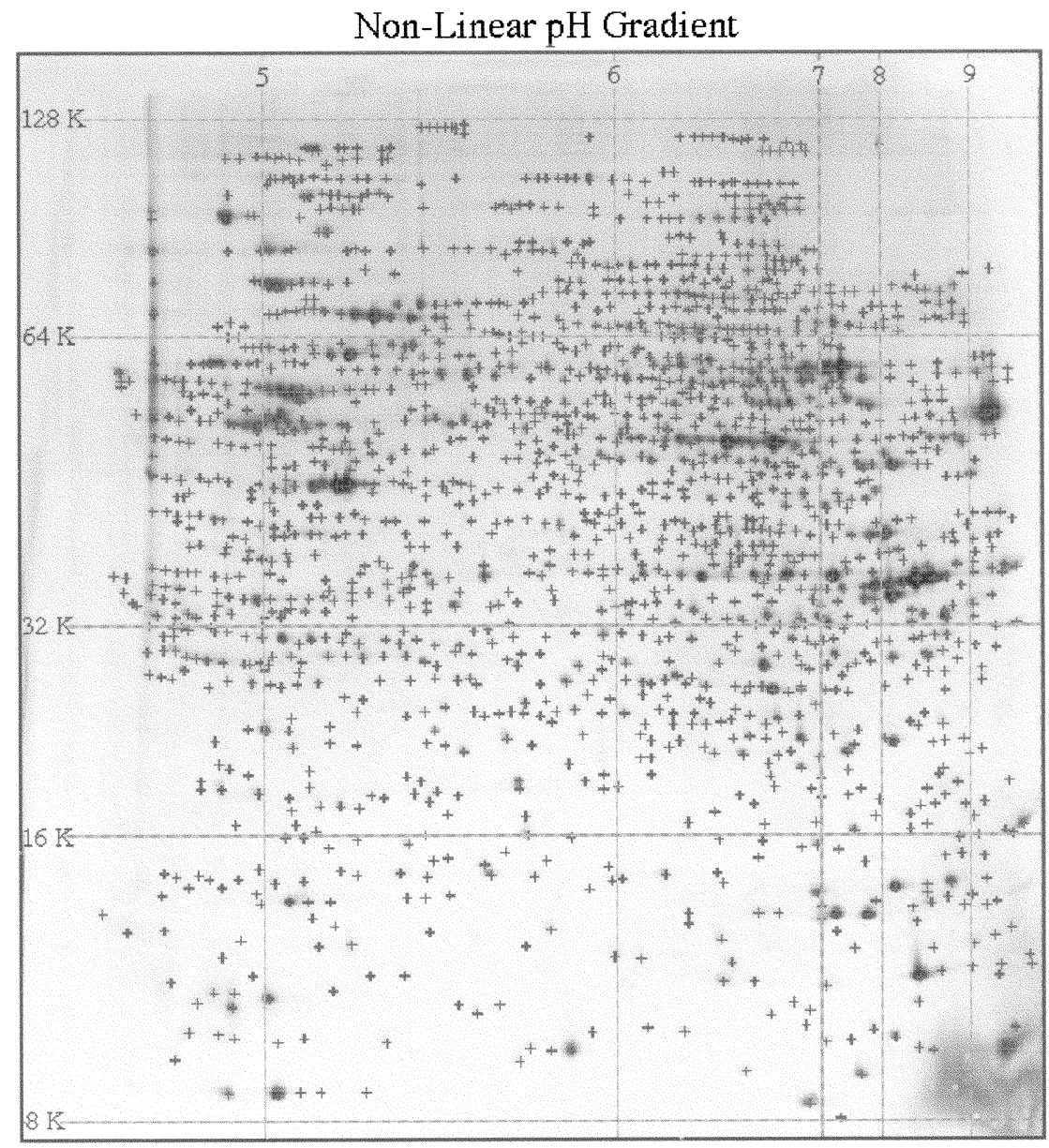

FIGURE 3. Representative two-dimensional electrophoretogram of proteins in the HB2 cell line matched with the 8701-BC profile.

group proteins involved in metabolism and biosynthesis, and the fourth group proteins and enzymes with regulatory functions. TABLES 1 to 4 list the protein groups, where proteins are indicated by the abbreviations used by the Swiss-Prot database. For each protein the accession number, $\mathrm{p}$, and $\mathrm{Mr}$ and the method of identification are given. When more isoforms of the same protein are present, alphabetical letters are added to the protein name abbreviation .

\section{Proteome Comparison between 8701-BC and HB2 Cells}

A representative number of 140 spots was arbitrarily selected to achieve unambiguous gel matching between the two maps. This number represents a significant 
TABLE 1. Cytoskeletal proteins

\begin{tabular}{lcccc}
\hline \multicolumn{1}{c}{ Protein } & $\mathrm{AC}$ & $\mathrm{pl}$ & $\mathrm{Mr}$ & $\mathrm{ID}$ method \\
\hline ACTB a & $\mathrm{P} 02570$ & 5.16 & 42,000 & $\mathrm{GM}$ \\
ACTB b & $\mathrm{P} 02570$ & 5.2 & 42,000 & $\mathrm{GM}$ \\
ACTB c & $\mathrm{P} 02570$ & 5.23 & 42,000 & $\mathrm{GM}$ \\
CK 8 & $\mathrm{P} 05787$ & $5.09-5.72$ & $42,000-49,830$ & $\mathrm{WB}$ \\
CK 18 & $\mathrm{P} 05783$ & $5.05-5.61$ & $47,074-51,800$ & $\mathrm{WB}$ \\
EZRI & $\mathrm{P} 15311$ & 6.04 & 75,194 & $\mathrm{GM}$ \\
TBB2 & $\mathrm{P} 05217$ & 4.98 & 49,913 & $\mathrm{GM}$ \\
\hline
\end{tabular}

TABLE 2. Folding, chaperones, and heat shock proteins

\begin{tabular}{lcccc}
\hline \multicolumn{1}{c}{ Protein } & AC & pl & Mr & ID method \\
\hline CBP2 a & P50454 & 9.23 & 49,309 & GM \\
CBP2 b & P50454 & 9.38 & 49,363 & GM \\
CRTC & P27797 & 4.52 & 59,300 & GM \\
CYPH a & P05092 & 6.66 & 14,500 & GM \\
CYPH b & P05092 & 7.07 & 14,500 & GM \\
CYPH c & P05092 & 7.51 & 14,500 & GM \\
ERP60 & P30101 & 5.88 & 53,600 & Nt-Ms \\
GRP 75 a & P38646 & 5.24 & 68,300 & GM \\
GRP 75 b & P38646 & 5.03 & 68,300 & GM \\
GRP 78 a & P11021 & 4.99 & 73,100 & GM \\
GRP 78 b & P11021 & 5.02 & 73,100 & GM \\
GRP 78 c & P11021 & 5.04 & 73,100 & GM \\
HSP 60 a & P10809 & 5.19 & 59,500 & GM \\
HSP 60 b & P10809 & 5.21 & 59,500 & GM \\
HSP 60 c & P10809 & 5.27 & 59,500 & GM \\
PDI & P07237 & 4.84 & 56,700 & GM \\
TCPZ & P40227 & 6.2 & 58,397 & GM \\
TRA & P14625 & 4.9 & 91,200 & GM \\
\hline
\end{tabular}

subset of proteins in the proteomic map, corresponding to about $8 \%$ of the total amount, and includes the proteins already identified and proteins with unknown identity (U), because they were absent from the data banks or N-terminal blocked. Their identification will be the object of further studies .

Triplicate experiments were performed to ensure reproducible protein separation; to exclude ambiguous spots, synthetic gels of the two cell preparations were constructed by means of the Melanie algorithms. FIGURE 4 shows the synthetic gel of the 8701-BC cell preparation and FIGURE 5 that obtained from HB2 cells. The iden- 
TABLE 3. Proteins involved in metabolism and biosynthesis

\begin{tabular}{lcccc}
\hline \multicolumn{1}{c}{ Protein } & AC & pl & Mr & ID method \\
\hline ALFA a & P04075 & 7.74 & 38,100 & GM \\
ALFA b & P04075 & 7.95 & 38,100 & GM \\
ENOA a & P06733 & 6.41 & 47,074 & WB \\
ENOA b & P06733 & 6.59 & 47,074 & WB \\
ENOA c & P06733 & 6.85 & 46,635 & WB \\
ENOA tr & $\ldots$ & 6.87 & 41,511 & Nt-Ms \\
G3P2 a & P04406 & 7.22 & 34,381 & Nt-Ms \\
G3P2 b & P04406 & 8.02 & 35,500 & Nt-Ms \\
G3P2 c & P04406 & 8.07 & 35,500 & Nt-Ms \\
G3P2 d & P04406 & 8.26 & 35,652 & Nt-Ms \\
G3P2 e & P04406 & 8.47 & 35,959 & Nt-Ms \\
G3P2 tr & $\ldots$ & 8.38 & 32,468 & Nt-Ms \\
KPY 1 & P14618 & 7.12 & 59,300 & GM \\
KPY 2 & P17786 & 7.28 & 59,300 & GM \\
TPIS a & P00938 & 6.51 & 25,000 & GM \\
TPIS b & P00938 & 6.81 & 25,000 & Nt-Ms \\
GSTP & P09211 & 6.01 & 21,054 & Nt-Ms \\
SODM & P04179 & 6.96 & 21,600 & GM \\
ACON & Q99798 & 6.91 & 79,190 & GM \\
MDHM & P40926 & 8.17 & 33,835 & Nt-Ms \\
MA32 & Q07021 & 4.54 & 28,600 & Nt-Ms \\
EF1B & P24534 & 4.62 & 29,200 & GM \\
EFTU & P49411 & 6.51 & 43,300 & Nt-Ms \\
RM12 & P52815 & 5.11 & 15,820 & GM \\
\hline
\end{tabular}

TABLE 4. Proteins and enzymes with regulatory functions

\begin{tabular}{lcccc}
\hline \multicolumn{1}{c}{ Protein } & AC & pl & Mr & ID method \\
\hline ANX1 & P04083 & 7.08 & 32,075 & Nt-Ms \\
ANX1-like & $\ldots$ & 7.89 & 26,932 & Nt-Ms \\
MIF & P14174 & 7.62 & 11,900 & GM \\
P53 & P04637 & 6.79 & 49,039 & GM \\
PSA5 & P28066 & 4.77 & 25,900 & Nt-Ms \\
TCTP & P13693 & 4.89 & 22,100 & GM \\
THIO & P10599 & 5.02 & 12,919 & Nt-Ms \\
UBIQ & P02248 & 6.89 & 8,700 & GM \\
UBL 1 & P09936 & 5.34 & 23,940 & Nt-Ms \\
\hline
\end{tabular}




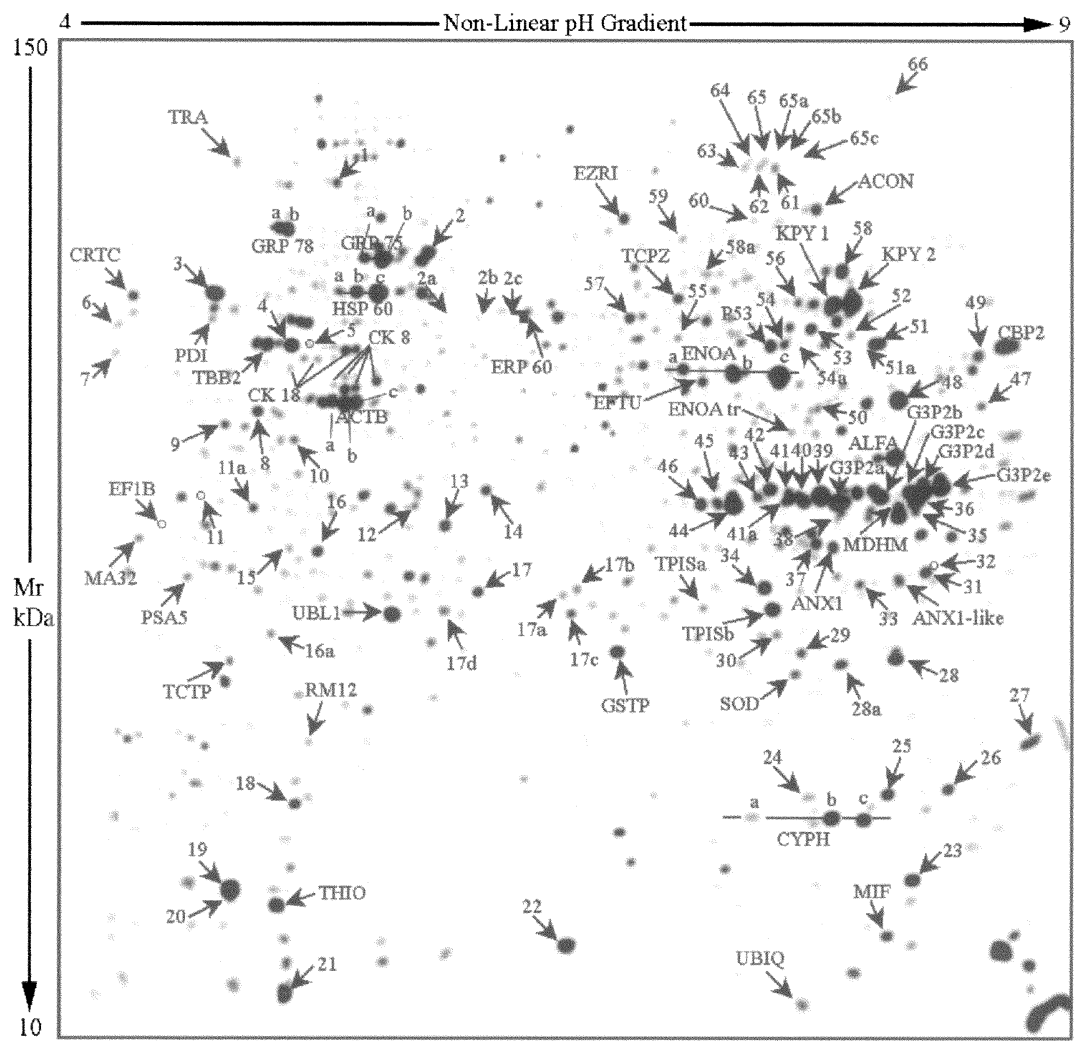

FIGURE 4. Synthetic gel created after elimination of background features from two-dimensional gels of 8701-BC cells. The proteins identified in triplicate gels are indicated with the abbreviations used in the Swiss-Prot database. The letters following the protein names indicate different isoforms. The numbers correspond to protein spots with unknown identity.

tified proteins are indicated with abbreviations used by the Swiss-Prot database; the spots corresponding to proteins with unknown identity are indicated with numbers.

Qualitative differences (presence/absence of spots) were observed within both groups of proteins with known and unknown identity. Proteins present in cancer cells and absent in the HB2 cell map were: the ubiquitin carboxyl-terminal hydrolase isozyme (UBL 1), an isoelectric variant of glutathione S-transferase (GSTP), and two proteins with unknown identity (spot numbers 35 and 36). Conversely, eight spots out of the panel of those selected (namely, 2a, 2b, 2c, 51a, 54, 65a, 65b, and $65 \mathrm{c}$ ) were uniquely present in the HB2 cell map. (Note that arrows point to corresponding positions of the expected spot, even in the absence of discernible stain.)

After qualitative analysis, we performed comparative quantitation of the comprehensive spectrum of selected protein spots, except the cytokeratins, which were identified by separate western blot analysis (not shown). The comparison was made by 


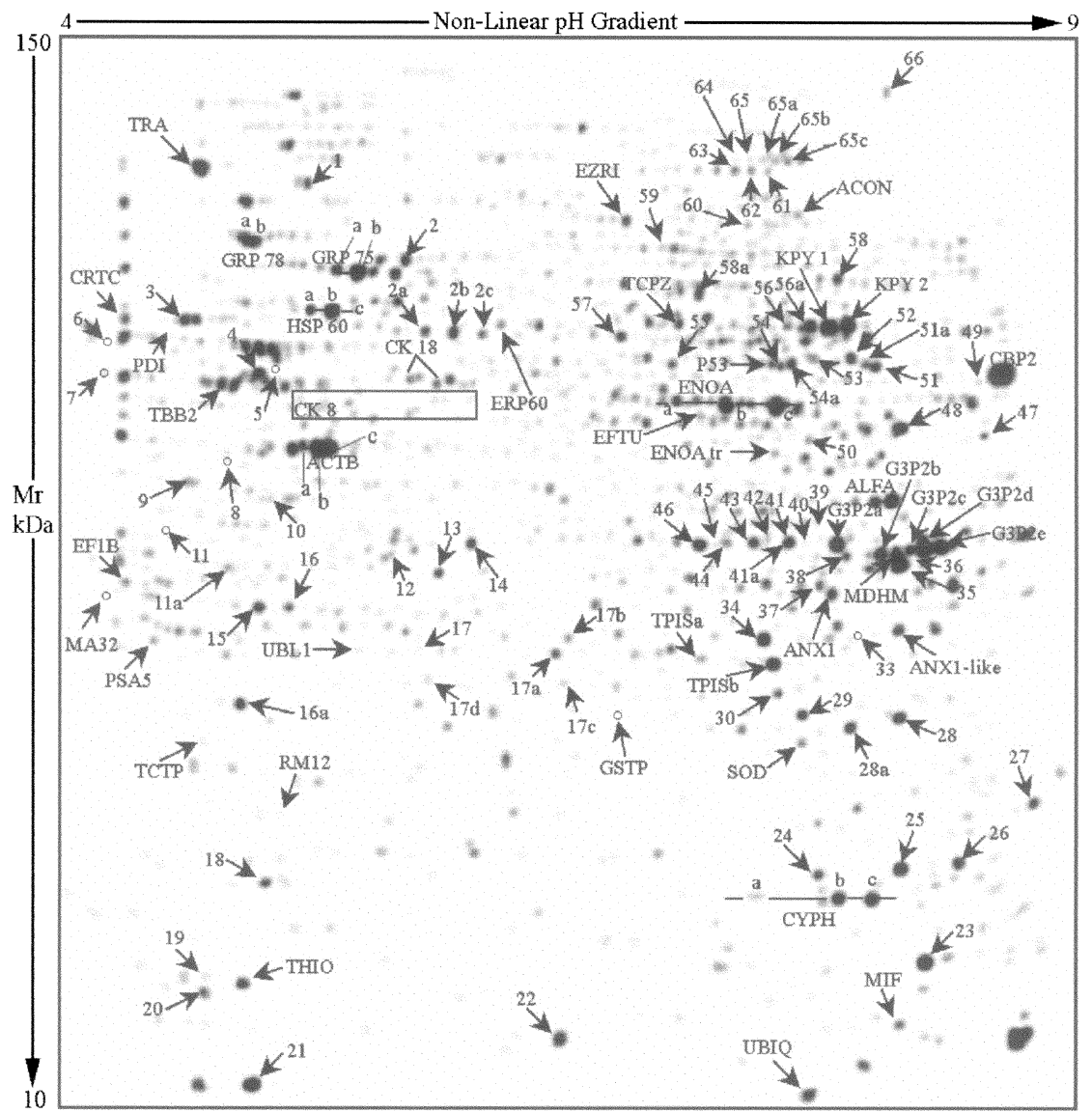

FIGURE 5. Synthetic gel created after elimination of background features from twodimensional gels of HB2 cells matched to the neoplastic cell profile. Note that in both maps some arrows point to corresponding positions of the expected spots, even in the absence of discernible stain.

plotting the relative abundance (\% Vol, average of triplicate experiments) of spots from the 8701-BC versus the corresponding spots of the HB2 cell map ordered according to increasing values (graphs 6 and 7) or vice versa (graph 8). The graph in FIGURE 6 shows the comparative profiles of known proteins, and the graphs in FIGURES 7 and 8 those of proteins with unknown identity.

As can be observed in the histograms, the profiles of the sampled protein are notably distinct in the two cell lines, because about $37 \%$ of the selected protein spots displayed variable levels of relative abundance in the two proteomes; in particular, 44 were overexpressed in cancer cells and 9 in epithelial cells, whereas the remainder displayed similar levels of expression in both maps. 


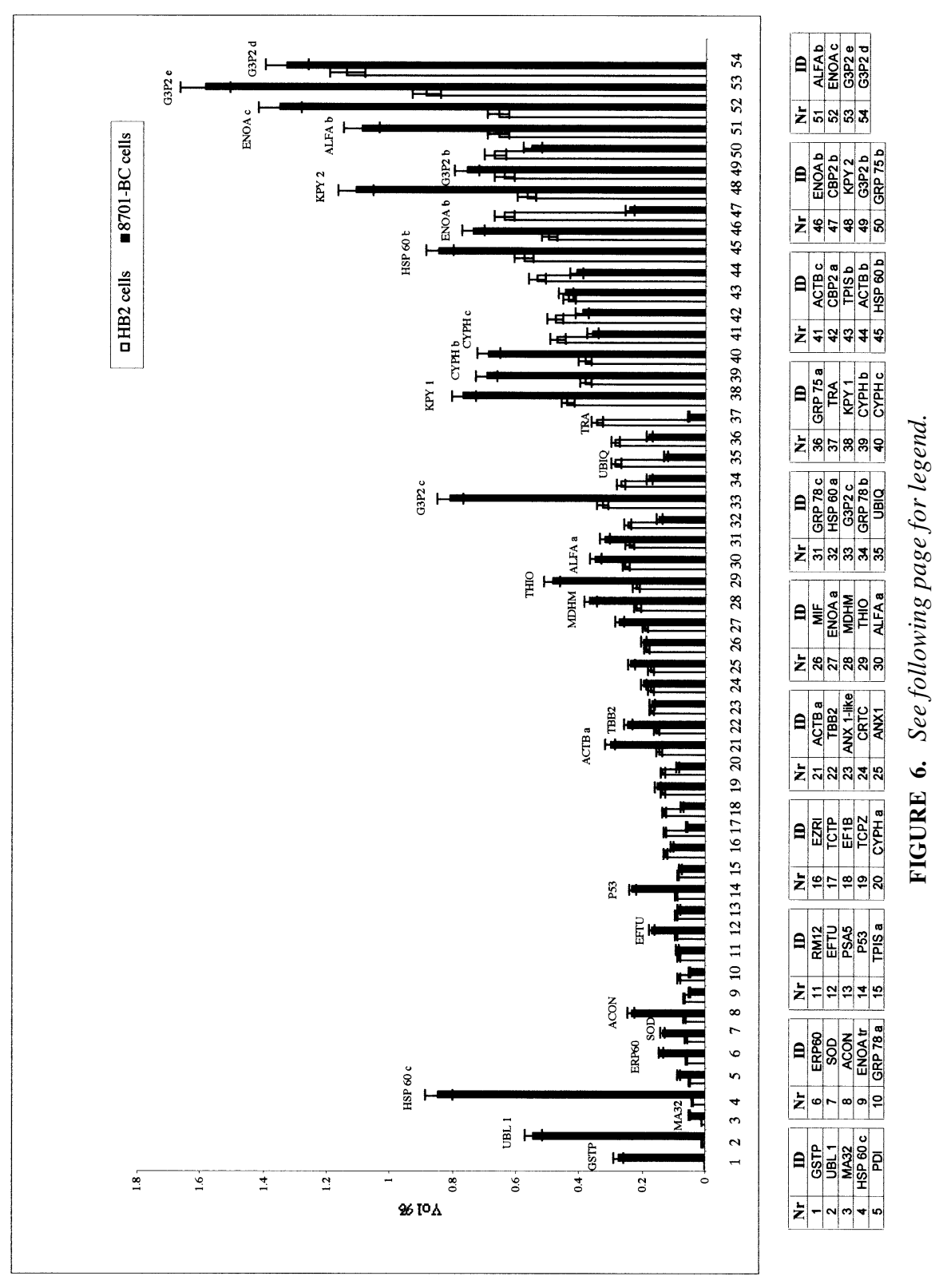


Proteins that showed higher intensity in cancer cells included glycolytic enzymes, chaperonins, and others with specific roles in biosynthesis and cellular metabolism. Conversely, no significant differences were found among the group of cytoskeletal proteins.

Among the glycolytic enzymes, glyceraldehyde-3-phosphate dehydrogenase (G3P2) showed an average increase of 1.5-fold relative to the control map. However, the various isoforms increased to a different extent, with the highest level of 2.5-fold exhibited by isoform c (TABLE 3). Similarly, alpha-enolase displayed an average increase of 1.7-fold, with the greatest difference for isoform c. An increment was also observed for fructose bisphosphate aldolase (1.37-fold for ALFA a and 1.5-fold for isoform b) and for pyruvate kinase (1.74-fold for KPY1 and 1.9-fold for KPY2). By contrast, no essential differences were observed for triosephosphate isomerase (TPIS).

Among the group of chaperonins, one isoform of mitochondrial molecular chaperone heat-shock protein 60 (HSP60) was found almost differentially present in cancer cells, whereas disulfide isomerase ER-60 (ERP 60) and peptidil prolyl cis-trans isomerase $(\mathrm{CYPH})$ showed increased levels of about twofold. A similar difference was found for elongation factor Tu (EFTU), thioredoxin (THIO), superoxide dismutase (SOD), oncosuppressor p53, and the MA32 glycoprotein, all proteins that play an active role in controlling cell proliferation and biosynthesis. Conversely, tumor rejection antigen 1 (TRA) and collagen binding protein (CBP2) were more expressed in HB2 cells.

Concerning proteins of unknown identity (marked $U$ followed by the number assigned in the 2D maps in FIGS. 2 and 3), two were uniquely present in cancer cells, as already mentioned (U35 and U36), and 22 were highly overexpressed (FIG. 7). The proteins overexpressed, or uniquely present, in epithelial cells relative to cancer cells are shown in FIGURE 8.

\section{DISCUSSION}

The proteomic strategy, based on two-dimensional gel electrophoresis and related techniques for protein identification and quantification, is a growing field of research in a world-wide dimension. However, comprehensive protein profiling in cancer occurs at its beginning. This work has contributed to the knowledge of breast cancer

FIGURE 6. Histogram showing a pattern of differentially expressed proteins between neoplastic and normal epithelial cells. Values in the ordinate represent the percentage of volume (integration of outer diameter [OD] over the feature's area) of individual spots over the sum of total spot volumes in each map. Bars indicate the standard deviation of triplicate measurements, which were 5-10\%. Correspondence between numbers $(\mathrm{Nr})$ in the abscissa and protein identity (ID) is given in the table below the graph.

FIGURE 7. Relative abundances (Vol\%) of a subset of protein spots with unknown identity, which are differentially expressed in normal and neoplastic cells. Graph highlights the protein spots more abundant in cancer cells relative to HB2 cells. The table below the graph lists the number of protein spots (corresponding to that assigned in the maps in FIGs. 5 and 6), with the $\mathrm{pI}$ and $\mathrm{Mr}$ coordinates. Standard deviation (not shown) of triplicate measurements was $5-10 \%$. 


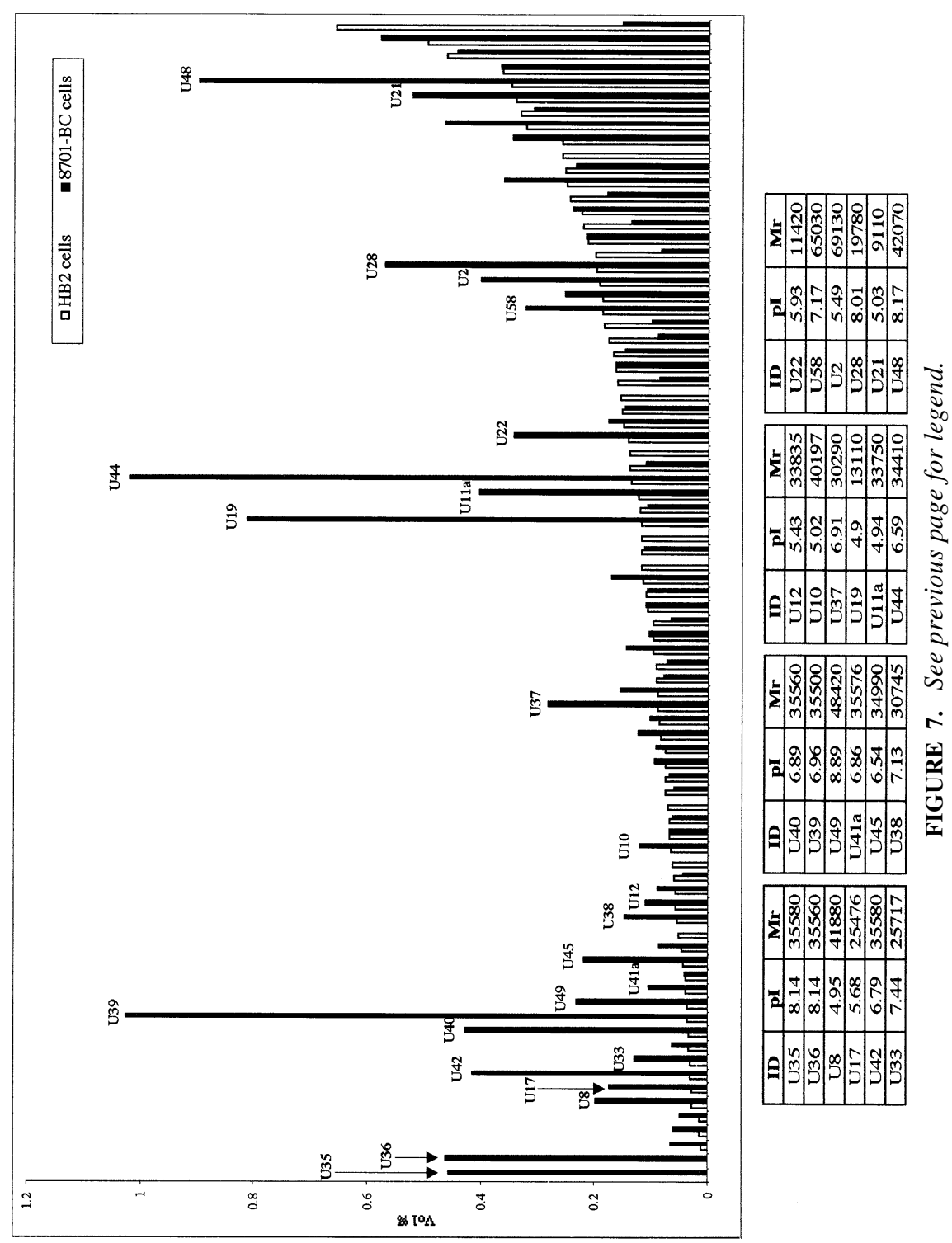




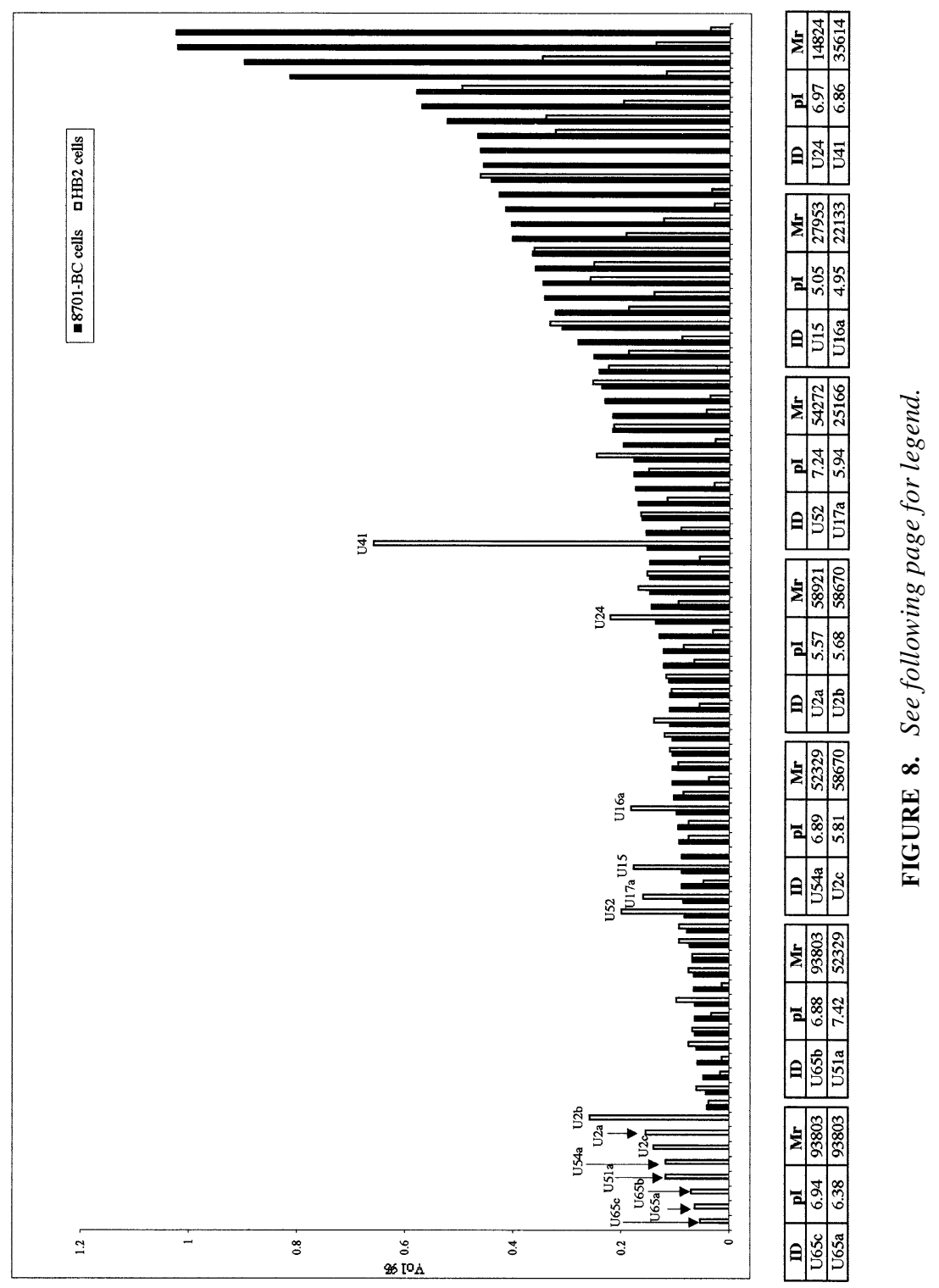


proteomics, using an in vitro model consisting of the well-characterized breast cancer cell line $8701-\mathrm{BC}^{5}$ and a human mammary epithelial cell line, HB2. ${ }^{7}$

Within the limitation of cultured cells, the two cell lines represent a suitable model, because each of them maintains in culture the specific features of their original in vivo status: $\mathrm{HB} 2$ cells display a polarized phenotype and are responsive to contact inhibition, whereas 8701-BC cells have pleomorphic morphology and overgrow, forming typical domes. Both of them express cytokeratin 8 and 18, which is typical of lumenal mammary cells. However, 8701-BC cells display isoelectric variants of the two cytokeratin forms, which are absent in normal cells (work in progress).

Within the experimental window of the 2D-IPG used, an average of 1,750 spots was detected by the Melanie system. To establish a significant area of overlapping between maps, we arbitrarily chose a subset of 140 representative spots, covering the $8 \%$ of the total protein complement in the maps. Fifty-eight proteins (including isoforms) were identified by gel matching with reference maps, immunodetection, or $\mathrm{N}$-terminal microsequencing and were classified according to their major functions. Of these, 9 belong to the class of cytoskeletal proteins, 18 to chaperonins and affine proteins, 23 are proteins involved in metabolism and biosynthesis, and 8 are involved in regulatory functions.

Results of computer-assisted matching of the neoplastic cell protein profile versus the normal epithelial cell profile demonstrated that within our selected protein sample, 12 proteins, four of which were uniquely present in the cancer cells, were differentially expressed in the two cell lines. Among these were isoelectric variants of glutathione S-transferase (GSTP) and the ubiquitin carboxyl-terminal hydrolase isozyme (UBL 1). The latter is a thiol protease involved in the processing of both ubiquitin precursors and ubiquinated proteins. It is interesting that this enzyme was originally found in neurons and cells of the diffuse neuroendocrine system and their tumors; therefore, its presence and function in breast cancer cells deserve further study.

In measuring changes in relative intensity within the subset of 140 protein spots, we found differences in about $37 \%$ of them, that is, 44 proteins were more elevated in cancer cells and 9 in epithelial cells.

Interestingly, most proteins overexpressed in cancer cells belong to the group of glycolytic enzymes. Elevated levels of anaerobic metabolism, even in the presence of oxygen, known as the "Warburg effect" after the scientist's discovery seven decades ago, are a frequent clinical syndrome in oncologic patients. ${ }^{13}$ However, the relative increment in glycolytic enzymes (or their isoforms) at the protein expression level was unexpected, because they are thought to be constitutively expressed by cells; in particular, G3P2 is used as one of the housekeeping genes to normalize transcriptional levels of other messengers in several experimental models.

Comparing our breast cancer cell map with that available on line (http://www.expasy.ch/ch2d/), we found that DIC tissues ${ }^{17}$ consistently and reproducibly show in-

FIGURE 8. Relative abundance of the same group of protein spots shown in the previous figure. The graph highlights a subset of protein spots more abundant in HB2 cells than in neoplastic cells. The table below shows the numbers of protein spots (corresponding to the maps in Figs. 5 and 6) with the pI and Mr coordinates. The standard deviation (not shown) of triplicate measurements was $5-10 \%$. 
creased levels of glycolytic enzymes in comparison with paired healthy tissues of the same patients. This observation allows us to conclude that the increased level of glycolytic enzymes in vivo is also maintained in cultured cancer cells and therefore may represent part of early oncogenic transformation in cancer rather than a mere adaptive response of tumor cells to the low oxygen supply in the host microenvironment.

This hypothesis is supported by recent molecular studies revealing that several of the multiple genetic alterations involved in tumor development directly affect glycolysis, which in turn increases the ability of tumor cells to recruit new blood vessel metabolism (Ref. 18 and references therein).

In conclusion, our current data have contributed to the knowledge of proteomic profiling of breast cancer cells. While indicating homologies with data already reported in vivo, they add new information on cancer-related proteomics, the knowledge of which will greatly enhance our understanding of molecular pathways involved in cancer development.

\section{ACKNOWLEDGMENTS}

This work was supported by AIRC and MURST (to I.P.M.). We thank Dr. Silvana Caricato (Centre of Experiemental OncoBiology, Palermo) for excellent technical assistance in cell culture preparation.

\section{REFERENCES}

1. Donegan, W.L. 1997. Tumor-related prognostic factors for breast cancer. Cancer J. Clin. 47: 28-51.

2. LANDER, E.S., L.M. Linton, B. BiRREN, et al. 2001. Initial sequencing and analysis of the human genome. Nature 409: 860-921.

3. Venter, J.C., M.D. AdAms, E.W. Myers, et al. 2001. The sequence of the human genome. Science 291: 1304-1351.

4. Emmert-Buck, M., J.P. GillesPie, C.P. ORnstein, et al. 2000. An approach to proteomic analysis of human tumors. Mol. Carcinog. 27: 158-165.

5. Minafra, S., V. Morello, F. Glorioso, et al. 1989. A new cell line (8701-BC) from primary ductal infiltrating carcinoma of human breast. Br. J. Cancer 60: 185-192.

6. Minafra, S., C. Giambelluca, M. Andriolo \& I. Pucci-Minafra. 1995. Cell-cell and cell-collagen interactions influence gelatinase production by human breast carcinoma cell line 8701-BC. Int. J. Cancer 62: 1-7.

7. BARTEK, J., J. BARTKOVA. N. KYPRIANOU, et al. 1991. Efficient immortalization of luminal epithelial cells from human mammary gland by introduction of simian virus 40 large tumor antigen with a recombinant retrovirus. Proc. Natl. Acad. Sci. USA 88: 3520-3524.

8. Berdichevsky, F., D. Alford, B. D'Souza \& J. Taylor-Papadimitriou. 1994. Branching morphogenesis of human mammary epithelial cells in collagen gels. J. Cell Sci. 107: 3557-3568.

9. Perou, C.M., S.S. JefFrey, M. van De Rijn, et al. 1999. Distinctive gene expression patterns in human mammary epithelial cells and breast cancers. Proc. Natl. Acad. Sci. USA 96: 9212-9217.

10. Williams, K., C. Chubb, E. Huberman \& C.S. Giometti. 1998. Analysis of differential protein expression in normal and neoplastic human breast epithelial cell lines. Electrophoresis 19: 333-343.

11. Görg, A., W. Postel \& S. Gunther. 1988. The current state of two dimensional electrophoresis with immobilized pH gradient. Electrophoresis 9: 531-546. 
12. Bjellqvist, B., C. Pasquali, F. Ravier, et al. 1993. A nonlinear wide-range immobilized $\mathrm{pH}$ gradient for two dimensional electrophoresis and its definition in a relevant pH scale. Electrophoresis 14: 1357-1365.

13. Hochstrasser, D.F., M.G. Harrington, A.C. Hochstrasser, et al. 1988. Methods for increasing the resolution of two dimensional protein electrophoresis. Anal. Biochem. 173: $424-435$.

14. Alessandro, R., S. Minafra, I. Pucci Minafra, et al. 1993. Metalloproteinase and Timp expression by the human breast carcinoma cell line 8701-BC. Int. J. Cancer 55: $250-255$.

15. Luparello, C., A. Noel \& I. Pucci-Minafra. 1997. Intratumoral heterogeneity for hsp90beta mRNA levels in a breast cancer cell line. DNA Cell Biol. 16: 1231-1236.

16. Luparello, C., A.F. Ginty, J.A. Gallagher, et al. 1993. TGF-beta, urokinase and PTHrP expression in 8701-BC breast cancer cells and clones. Differentiation 55: $73-$ 80.

17. Bini, L., B. Magi, B. Marzocchi, et al. 1997. Protein expression profiles in human breast ductal carcinoma and histologically normal tissue. Electrophoresis 18: $2832-$ 2841.

18. Dang, C.V. \& G.L. Semenza. 1999. Oncogenic alterations of metabolism. Trends Biochem. Sci. 24: 68-72. 